\title{
RIEMANNIAN SUBMERSIONS NEED NOT PRESERVE POSITIVE RICCI CURVATURE
}

\author{
CURTIS PRO AND FREDERICK WILHELM
}

(Communicated by Lei $\mathrm{Ni}$ )

\begin{abstract}
If $\pi: M \rightarrow B$ is a Riemannian submersion and $M$ has positive sectional curvature, O'Neill's Horizontal Curvature Equation shows that $B$ must also have positive curvature. We show there are Riemannian submersions from compact manifolds with positive Ricci curvature to manifolds that have small neighborhoods of (arbitrarily) negative Ricci curvature, but that there are no Riemannian submersions from manifolds with positive Ricci curvature to manifolds with nonpositive Ricci curvature.
\end{abstract}

In [2], O'Neill shows that if $\pi: M \rightarrow B$ is a Riemannian submersion, then

$$
\sec _{B}(x, y)=\sec _{M}(\tilde{x}, \tilde{y})+3\left|A_{\tilde{x}} \tilde{y}\right|^{2},
$$

where $\{x, y\}$ is an orthonormal basis for a tangent plane to $B,\{\tilde{x}, \tilde{y}\}$ a horizontal lift of $\{x, y\}$ and $A$ is O'Neill's $A$-tensor. An immediate observation is that if the sectional curvature of all two-planes in $M$ are bounded below by $k$, then the same must be true for all two-planes in $B$. In particular, Riemannian submersions preserve positive sectional curvature.

David Wraith asked us for an example of a Riemannian submersion that does not preserve positive Ricci curvature. While many people think that such an example must exist, we do not know of one in the literature but provide some here.

Theorem 1. For any $C>0$, there is a Riemannian submersion $\pi: M \rightarrow B$ for which $M$ is compact with positive Ricci curvature and $B$ has some Ricci curvatures less than $-C$.

The examples are constructed as warped products $S^{2} \times{ }_{\nu} F$, where $F$ is any manifold that admits a metric with Ricci curvature $\geq 1$, and the metrics on $S^{2}$ are $C^{1}$-close to the constant curvature 1 metric. In his thesis, the first author provides examples where the base metric on $S^{2}$ can be $C^{1}$-close to any predetermined, positively-curved, rotationally-symmetric metric on $S^{2}$, and $F$ is any manifold that admits a metric with Ricci curvature $\geq 1$ [7].

Since the metrics on our base spaces are $C^{1}$-close to the constant curvature 1 metric on $S^{2}$ and yet have a curvature that is $\leq-C$, the region of negative curvature is necessarily small, and in fact has measure converging to 0 as $-C \rightarrow-\infty$. In this sense, our examples are local. On the other hand, we show that a truly global example is not possible.

Received by the editors June 17, 2012 and, in revised form, July 12, 2012.

2010 Mathematics Subject Classification. Primary 53C20.

(C)2014 American Mathematical Society Reverts to public domain 28 years from publication 
Theorem 2. If $M$ is a compact Riemannian manifold with positive Ricci curvature, then there is no Riemannian submersion $\pi: M \longrightarrow B$ to a space $B$ with nonpositive Ricci curvature.

We prove Theorem 1 in Sections 1 and 2 and Theorem 2 in Section 3.

\section{VERTICAL WARPING}

Given a Riemannian submersion $\pi: M \rightarrow B$, the vertical and horizontal distributions are defined as $\mathcal{V}:=\operatorname{ker} \pi_{*}$ and $\mathcal{H}:=\left(k e r \pi_{*}\right)^{\perp}$, respectively. This gives a splitting of the tangent bundle as

$$
T M=\mathcal{V} \oplus \mathcal{H} .
$$

If $g$ is the metric on $M$, we denote by $g^{h}$ and $g^{v}$ the restrictions of $g$ to $\mathcal{H}$ and $\mathcal{V}$. Define a new metric $g_{\nu}:=e^{2 \nu} g^{v}+g^{h}$ on $M$, where $\nu$ is any smooth function on $B$. Note that both $\mathcal{H}$ and $g^{h}$ are unchanged, so $\pi:\left(M, g_{\nu}\right) \rightarrow B$ is also Riemannian.

The calculations that give important geometric quantities associated to $g_{\nu}$ in terms of $g$ and $\nu$ are carried out in [1] on page 45. In particular, the $(0,2)$ Ricci tensor $\operatorname{Ric}_{\nu}$ of $g_{\nu}$ is given in detail. When $M=B^{m} \times F^{k}$ and $g$ is a product metric, these quantities reduce to the following (Corollary 2.2.2 of [1]).

For horizontal $X, Y$ and vertical $U, V$, we have

(1.1) $\operatorname{Ric}_{\nu}(X, Y)=\operatorname{Ric}_{B}(X, Y)-k(\operatorname{Hess} \nu(X, Y)+g(\nabla \nu, X) g(\nabla \nu, Y))$,

(1.2) $\operatorname{Ric}_{\nu}(X, U)=0$, and

(1.3) $\operatorname{Ric}_{\nu}(U, V)=\operatorname{Ric}_{F}(U, V)-g(U, V) e^{2 \nu}\left(\Delta \nu+k|\nabla \nu|^{2}\right)$.

(There is a sign error in the analog of Equation 1.3 in [1].) We denote fields by $B$ and their horizontal lifts via $\pi_{1}: B \times F \rightarrow B$ by the same letter. We write $B \times{ }_{\nu} F$ to denote the warped product metric $g_{\nu}$ on $B \times F$.

\section{The WARPEd PRoduct $S_{\varphi}^{2} \times{ }_{\nu} F$}

Choose $\varphi:[0, \pi] \rightarrow[0, \infty)$ so that $S^{2}$ with the metric $g_{\varphi}=d r^{2}+\varphi^{2} d \theta^{2}$ is a smooth Riemannian manifold denoted by $S_{\varphi}^{2}$. Let $\nu:[0, \pi] \rightarrow R$ be a function on $S_{\varphi}^{2}$ that only depends on $r$. Consider the warped product $S_{\varphi}^{2} \times{ }_{\nu} F$, where $\left(F, g_{F}\right)$ is any $k$-dimensional manifold $(k \geq 2)$ with $\operatorname{Ric}_{F} \geq 1$. Using the notation $\dot{\nu}=\partial_{r} \nu$, since $\nu$ only depends on $r$,

$$
\nabla \nu=\dot{\nu} \partial_{r}
$$

If $L$ is the Lie derivative, we have

$$
\begin{aligned}
2 \operatorname{Hess} \nu & =L_{\nabla \nu} g_{\varphi} \\
& =L_{\dot{\nu} \partial_{r}} g_{\varphi} \\
& =\dot{\nu} L_{\partial_{r}} g_{\varphi}+d \dot{\nu} d r+d r d \dot{\nu} \\
& =2 \dot{\nu} \operatorname{Hess} r+2 \ddot{\nu} d r^{2} .
\end{aligned}
$$

Thus the Hessian of $\nu$ is given by

$$
\text { Hess } \nu=\ddot{\nu} d r^{2}+\dot{\nu} \varphi \dot{\varphi} d \theta^{2} \text {. }
$$


The Ricci tensor of $S_{\varphi}^{2}$ is given as

$$
\operatorname{Ric}_{S_{\varphi}^{2}}=-\frac{\ddot{\varphi}}{\varphi} g_{\varphi}
$$

(see 4], p. 69). Let $\operatorname{Ric}_{\nu}^{h}$ and $\operatorname{Ric}_{\nu}^{v}$ denote $\operatorname{Ric}_{\nu}$ restricted to the horizontal and vertical distribution, respectively. Equation (1.1) can be written as

$$
-\operatorname{Ric}_{\nu}^{h}=\left[\frac{\ddot{\varphi}}{\varphi}+k\left(\ddot{\nu}+\dot{\nu}^{2}\right)\right] d r^{2}+\varphi[\ddot{\varphi}+k \dot{\nu} \dot{\varphi}] d \theta^{2},
$$

and equation (1.3) can be written as

$$
\operatorname{Ric}_{\nu}^{v}=\operatorname{Ric}_{F}-e^{2 \nu}\left(\ddot{\nu}+\frac{\dot{\varphi} \dot{\nu}}{\varphi}+k \dot{\nu}^{2}\right) g_{F} .
$$

Notice that since $\operatorname{Ric}_{F} \geq 1$, if $\operatorname{Ric}_{\nu}^{h}$ is positive, then these equations together with equation (1.2) imply that $S_{\varphi}^{2} \times{ }_{\nu+\ln \lambda} F$ has positive Ricci curvature, provided $\lambda$ is a sufficiently small positive constant.

By requiring that $\ddot{\varphi}(p)>0$ for some point $p \in(0, \pi)$, the projection $\pi_{1}: S_{\varphi}^{2} \times{ }_{\nu}$ $F \longrightarrow S_{\varphi}^{2}$ is a Riemannian submersion for which the base has points of negative Ricci curvature. Therefore, to describe a Riemannian submersion that does not preserve positive Ricci curvature, it suffices to find functions $\varphi$ and $\nu$ so that

(1) $S_{\varphi}^{2}$ is smooth and has points of negative curvature, that is,

$$
\ddot{\varphi}(p)=\eta>0
$$

for some point $p \in(0, \pi)$,

(2) $\operatorname{Ric}_{\nu}^{h}>0$, that is,

$$
\begin{aligned}
\ddot{\nu}+\dot{\nu}^{2} & <-\frac{\ddot{\varphi}}{k \varphi}, \\
\dot{\varphi} \dot{\nu} & <-\frac{\ddot{\varphi}}{k},
\end{aligned}
$$

and

(3) $\nu$ is constant in a neighborhood of 0 and $\pi$.

For a $C^{r}$-function $f: R \longrightarrow R$, we write

$$
\|f\|_{C^{r}}:=\sup _{k \leq r} \sup _{x \in \mathbb{R}}\left\{\left|f^{(k)}(x)\right|\right\} .
$$

The main idea is embodied in the following lemma.

Lemma 1. Given any sufficiently small $\eta>0, p \in(0, \pi / 4)$, and $\varepsilon>0$, there is a $\delta>0$ and smooth functions $\varphi, \nu:[0, \pi] \rightarrow[0, \infty)$ with the following properties:

(1) On $[0, \pi] \backslash(p-\delta, p+\delta)$,

$$
\|\varphi-\sin \|_{C^{2}}<\varepsilon
$$

and $\ddot{\varphi}$ is maximal at $p$ with $\ddot{\varphi}(p)=\eta>0$.

(2) On $[0, \pi]$,

$$
\|\varphi-\sin \|_{C^{1}}<\varepsilon,
$$

and for all $r$ in a small neighborhood about the endpoints of $[0, \pi]$,

$$
\varphi(r)=\sin (r) .
$$

(3) $\nu$ is constant in a neighborhood of 0 and $\pi$. 
(4) On $(p-2 \delta, p+\delta)$,

$$
\begin{aligned}
\ddot{\nu}+\dot{\nu}^{2} & <-\frac{\ddot{\varphi}}{k \varphi} \text { and } \\
\dot{\nu} \dot{\varphi} & <-\frac{\ddot{\varphi}}{k} .
\end{aligned}
$$

(5) On $[0, \pi] \backslash(p-2 \delta, p+\delta)$,

$$
\|\nu\|_{C^{2}} \leq 20 \frac{\eta}{k}
$$

Proof. By controlling the size of the interval for which $\ddot{\varphi}$ is very different from - sin, one can construct a function $\varphi$ that satisfies (1) and (2), by prescribing $\ddot{\varphi}$ and integrating.

Since $\|\varphi-\sin \|_{C^{2}}$ is small on $[0, \pi] \backslash(p-\delta, p+\delta),(4)$ is satisfied on $[0, p-\delta]$, provided

$$
\begin{aligned}
\left.\nu\right|_{[0, p-2 \delta]} & \equiv 0 \text { and } \\
\left.\left(\ddot{\nu}+\dot{\nu}^{2}\right)\right|_{(p-2 \delta, p-\delta]} & <0 .
\end{aligned}
$$

Since $\ddot{\varphi}$ is maximal at $p$ with $\ddot{\varphi}(p)=\eta>0$, and since $\|\varphi-\sin \|_{C^{1}}<\varepsilon$, (4) is satisfied by $[p-\delta, p+\delta]$, provided

$$
\begin{aligned}
\left.\left(\ddot{\nu}+\dot{\nu}^{2}\right)\right|_{[p-\delta, p+\delta]} & <-2 \frac{\eta}{k p} \text { and } \\
\frac{\left.\dot{\nu}\right|_{[p-\delta, p+\delta]}}{\sqrt{2}} & <-2 \frac{\eta}{k} .
\end{aligned}
$$

We require that $\eta$ be small enough so that $\left(\frac{\eta}{k}\right)^{2}<\frac{\eta}{100 k p}$. By prescribing $\ddot{\nu}$ and integrating, we can then choose $\nu$ so that it satisfies (2.3) on $[0, p-\delta]$, (2.4) on $[p-\delta, p+\delta]$, and, in addition,

$$
\begin{aligned}
\dot{\nu}(p-\delta) & =-3 \frac{\eta}{k} \text { and } \\
-4 \frac{\eta}{k p} & \leq\left.\ddot{\nu}\right|_{[p-\delta, p+\delta]}<0 .
\end{aligned}
$$

We then have the following bound on $\dot{\nu}(p+\delta)$ :

$$
\begin{aligned}
0 & >\dot{\nu}(p+\delta) \\
& =\dot{\nu}(p-\delta)+\int_{p-\delta}^{p+\delta} \ddot{\nu} \\
& \geq-3 \frac{\eta}{k}-2 \delta\left(4 \frac{\eta}{k p}\right) .
\end{aligned}
$$

So by choosing $\delta$ to be sufficiently small, we can, by prescribing $\ddot{\nu}$, extend $\nu$ to satisfy (5) and (3) on $[p+\delta, \pi]$.

By composing with projection, $\pi_{1}: S^{2} \times F \longrightarrow S^{2}$, we can view $r$ as a function on $S^{2} \times F$, which by abuse of notation we also call $r$.

Proposition 1. There is an $\eta>0$ with the following property. For any $p \in\left(0, \frac{\pi}{4}\right)$, there is an $\varepsilon(p)>0$ so that for $\varphi$ and $\nu$ as in Lemma 1,

$$
\operatorname{Ric}_{\nu}^{h} \geq 1 / 2
$$

on $r^{-1}([0, \pi] \backslash(p-2 \delta, p+\delta))$. 
Proof. On $r^{-1}([0, \pi] \backslash(p-2 \delta, p+\delta))$, we have $\|\varphi-\sin \| C_{C^{2}}<\varepsilon$ and $\|\nu\|_{C^{2}}<$ $20 \pi \frac{\eta}{k}$. Since $\operatorname{Ric}_{F} \geq 1$, we have $\operatorname{Ric}\left(S^{2}(1) \times F\right) \geq 1$. Combining these facts with

$$
\operatorname{Ric}_{\nu}^{h}=-\left[\frac{\ddot{\varphi}}{\varphi}+k\left(\ddot{\nu}+\dot{\nu}^{2}\right)\right] d r^{2}-\varphi[\ddot{\varphi}+k \dot{\nu} \dot{\varphi}] d \theta^{2}
$$

gives the result.

Proof of Theorem 1. Let $\eta>0$ be as in Proposition 11. Given $C>0$, choose $p \in\left(0, \frac{\pi}{4}\right)$ so that

$$
-\frac{\eta}{2 p}<-C
$$

Choose $\varphi$ and $\nu$ as in Proposition 1, Then

$$
\operatorname{Ric}_{\nu}^{h} \geq 1 / 2
$$

on $r^{-1}([0, \pi] \backslash(p-2 \delta, p+\delta))$, and by part (4) of Lemma1] $\operatorname{Ric}_{\nu}^{h}>0$ on $r^{-1}(p-2 \delta$, $p+\delta)$. It follows that $\operatorname{Ric}_{\nu}>0$ everywhere.

On the other hand, along $r^{-1}(p)$, the Ricci curvature of $S_{\varphi}^{2}$ is

$$
\operatorname{Ric}_{S_{\varphi}^{2}}=-\frac{\ddot{\varphi}(p)}{\varphi(p)} g_{\varphi}
$$

Since $\ddot{\varphi}(p)=\eta$ and $\varphi(p)$ is almost $\sin (p)$,

$$
\operatorname{Ric}_{S_{\varphi}^{2}} \leq-\frac{\eta}{2 p}<-C
$$

as desired.

Remark 1. The proof above exploits the principle of Lemma 3.1 of [5] where components of the curvature change a lot under a certain type of $C^{1}$-small deformation. This principle will be applied in a revised version [6], where a revised version of Lemma 3.1 of [5] will appear.

\section{The Base has a POSItive Ricci CuRvature}

In this section, we prove Theorem 2 with an argument that is similar in spirit to the proof of the main theorem of [3]. We use the notation of [2] for the infinitesimal geometry of a submersion.

Suppose $\pi: M \longrightarrow B$ is a Riemannian submersion with $M$ compact, $\operatorname{Ric}_{M}>0$ and $\operatorname{Ric}_{B} \leq 0$. Exploiting the compactness of the unit tangent bundle $T^{1} M$ of $M$, we get Propositions 2 and 3 , stated below. To pose them we need a metric space structure on $T^{1} M$. Any metric that induces the topology of $T^{1} M$ will suffice; the most geometrically relevant is perhaps the Sasaki metric (see [8]).

Proposition 2. Let $\left(T^{1} M\right.$, dist) be the unit tangent bundle of $M$ with any metric distance that induces the topology of $T^{1} M$. For any $\varepsilon>0$, there is a unit speed, horizontal geodesic $\gamma:[0, l] \longrightarrow M$ so that $l \geq 1$ and

$$
\operatorname{dist}(\dot{\gamma}(0), \dot{\gamma}(l))<\varepsilon .
$$

Proof. Let $\gamma: \mathbb{R} \longrightarrow M$ be any unit speed horizontal geodesic. Since $T^{1} M$ is compact, the infinite set $\{\dot{\gamma}(n)\}_{n=1}^{\infty}$ has an accumulation point. In particular, there are integers $j, k$ so that

$$
\operatorname{dist}(\dot{\gamma}(j), \dot{\gamma}(k))<\varepsilon .
$$

Reparameterizing produces the desired geodesic. 
Proposition 3. Let $\mathcal{H}^{1}$ be the set of unit horizontal vectors for $\pi$. The map $\mathcal{T}$ : $\left(\mathcal{H}^{1}\right.$, dist $) \longrightarrow R$ defined by

$$
\mathcal{T}(x)=\operatorname{Trace}\left(V \longmapsto T_{V} x\right)
$$

is uniformly continuous. Here $T$ is O'Neill's T-tensor.

Proof of Theorem 2. Let $\gamma$ be a horizontal, almost periodic geodesic as in Proposition 2. By re-scaling we may assume that $\operatorname{Ric}_{M} \geq 1$. Then

$$
l \leq \int_{0}^{l} \operatorname{Ric}(\dot{\gamma}, \dot{\gamma}) .
$$

Let $\left\{V_{i}\right\}_{i=1}^{k}$ be a vertically-parallel, orthonormal framing for the vertical distribution along $\gamma$, and let $\left\{E_{j}\right\}_{j=1}^{b}$ be an orthonormal framing for the horizontal distribution along $\gamma$. Using O'Neill's vertical and horizontal curvature equations and the hypothesis that $\operatorname{Ric}_{B} \leq 0$, we get

$$
\begin{aligned}
l \leq & \int_{0}^{l} \operatorname{Ric}(\dot{\gamma}, \dot{\gamma}) \\
= & \sum_{i} \int_{0}^{l} D_{\dot{\gamma}}\left\langle T_{V_{i}} V_{i}, \dot{\gamma}\right\rangle-\left|T_{V_{i}} \dot{\gamma}\right|^{2}+\left|A_{\dot{\gamma}} V_{i}\right|^{2} \\
& +\sum_{j} \int_{0}^{l} \operatorname{Ric}_{B}(\dot{\gamma}, \dot{\gamma})-3\left|A_{\dot{\gamma}} E_{j}\right|^{2} \\
\leq & \sum_{i}\left(\left.\left\langle T_{V_{i}} V_{i}, \dot{\gamma}\right\rangle\right|_{0} ^{l}+\int_{0}^{l}\left|A_{\dot{\gamma}} V_{i}\right|^{2}\right)+\sum_{j} \int_{0}^{l}-3\left|A_{\dot{\gamma}} E_{j}\right|^{2} .
\end{aligned}
$$

Since $\left\{V_{i}\right\}_{i=1}^{k}$ and $\left\{E_{j}\right\}_{j=1}^{b}$ are orthonormal frames,

$$
\begin{aligned}
\sum_{j}\left|A_{\dot{\gamma}} E_{j}\right|^{2} & =\sum_{j} \sum_{i}\left\langle A_{\dot{\gamma}} E_{j}, V_{i}\right\rangle^{2} \\
& =\sum_{i} \sum_{j}\left\langle E_{j}, A_{\dot{\gamma}} V_{i}\right\rangle^{2} \\
& =\sum_{i}\left|A_{\dot{\gamma}} V_{i}\right|^{2} .
\end{aligned}
$$

Therefore (3.1) gives us

$$
\begin{aligned}
1 & \leq l \leq \sum_{i}\left(\left.\left\langle T_{V_{i}} V_{i}, \dot{\gamma}\right\rangle\right|_{0} ^{l}\right) \\
& =\sum_{i}-\left.\left\langle V_{i}, T_{V_{i}} \dot{\gamma}\right\rangle\right|_{0} ^{l} \\
& =\left.\operatorname{Trace}\left(W \longmapsto-T_{W} \dot{\gamma}\right)\right|_{0} ^{l} .
\end{aligned}
$$

From Propositions 2 and 3 , we can choose a horizontal $\gamma:[0, l] \longrightarrow M$ so that

$$
\left.\operatorname{Trace}\left(W \longmapsto-T_{W} \dot{\gamma}\right)\right|_{0} ^{l}<\frac{1}{2},
$$

which gives a contradiction. 


\section{ACKNOWLEDGMENTS}

The authors are grateful to the referee for noticing and fixing a shortcoming in Proposition 2, to Bun Wong for asking a question that led them to Theorem 2, to Pedro Solórzano for assisting them with a calculation, and especially to David Wraith for insightful criticisms of the manuscript and for asking the authors to provide the examples of Theorem [1.

\section{REFERENCES}

[1] Detlef Gromoll and Gerard Walschap, Metric foliations and curvature, Progress in Mathematics, vol. 268, Birkhäuser Verlag, Basel, 2009. MR2500106 (2010h:53035)

[2] Barrett O'Neill, The fundamental equations of a submersion, Michigan Math. J. 13 (1966), 459-469. MR0200865 (34 \#751)

[3] Ye-Lin $\mathrm{Ou}$ and Frederick Wilhelm, Horizontally homothetic submersions and nonnegative curvature, Indiana Univ. Math. J. 56 (2007), no. 1, 243-261, DOI 10.1512/iumj.2007.56.2999. MR2305936 (2009b:53042)

[4] P. Petersen, Riemannian geometry, 2nd ed., Graduate Texts in Math., 171, Springer, New York, 2006. MR2243772

[5] P. Petersen and F. Wilhelm, Principles for deforming nonnegative curvature, preprint, http://arxiv.org/pdf/0908.3026.pdf.

[6] P. Petersen and F. Wilhelm, An exotic sphere with positive sectional curvature, preprint, http://arxiv.org/pdf/0805.0812v3.pdf.

[7] C. Pro, On Riemannian submersions and diffeomorphism stability, Ph.D. thesis, University of California, Riverside, 2012.

[8] Shigeo Sasaki, On the differential geometry of tangent bundles of Riemannian manifolds, Tôhoku Math. J. (2) 10 (1958), 338-354. MR0112152 (22 \#3007)

Department of Mathematics, University of California, Riverside, Riverside, CaliFORNIA 92521

Current address: Department of Mathematics, University of Toronto, Toronto, ON, Canada M5S 2E4

E-mail address: cpro@math.toronto.edu

Department of Mathematics, University of California, Riverside, Riverside, CaliFORNIA 92521 